Proc. VII National Conference of Neutron Scattering and Complementary Methods, Chlewiska, Poland, June 12-16, 2011

\title{
Melting as an Instability of Crystal Structure with respect to Transverse Acoustic Wave, Quasi-Harmonic Approach
}

\author{
A. $\mathrm{CzACHOR}^{*}$ \\ National Center for Nuclear Research, Świerk, 05-400 Otwock, Poland
}

\begin{abstract}
It is suggested that the precursor of melting in some crystals can be a macroscopic shear deformation such as the transverse sound wave. This hypothesis is examined basing on the quasi-harmonic approach to lattice dynamics within the two-body ion-ion concept of interaction in metals. One finds that due to the temperature induced modification of effective ion-ion interaction appearing in the quasi-harmonic approximation the crystal ceases to support propagation of transverse sound waves when its temperature approaches a certain critical temperature $T_{\mathrm{cr}}$, understood here to be the melting temperature $T_{\mathrm{m}}$.
\end{abstract}

PACS: $64.70 . d j$

\section{Introduction}

One may ask the question - what is the initial (primordial) deformation of the crystal structure caused by an increase of temperature, which destroys its periodic order turning it finally into a liquid?

Melting of a solid is the two-phase phenomenon - at melting temperature $T_{\mathrm{m}}$ the solid and the liquid coexist, see e.g. Ubbelohde [1]. Contemporary physical theory allows one to approximately predict the dependence of $T_{\mathrm{m}}$ on hydrostatic pressure $p$ - the melting curve $T_{\mathrm{m}}(p)-$ and other characteristics [2], using the statistical $a b$ initio methods for both phases independently [3]. At the same time the one-phase theory comparing the average atomic displacement $\sqrt{\left\langle u^{2}\right\rangle}$ to the inter-atomic distance in crystal - the Lindeman theory - also offers quite universal understanding of melting phenomena, see $[4,5]$ and references quoted there. Still one expects that there may be a specific initial deformation which appears, magnifies and tends to destroys periodic order, when the crystal approaches the melting temperature. Of course, such pivotal deformations may be different in different materials.

The aim of this paper is the theoretical examination of one such specific deformation of metal crystal structure close to the melting temperature. Our hypothesis is that the melting can be connected to a shear deformation of crystal below $T_{\mathrm{m}}$, see Fig. 1, such as is present in the transverse sound wave. This should manifest itself in a critical dependence on temperature of the corresponding displacement-displacement correlation function, and this object can be calculated.

Experimental data show that all elastic constants $c_{i j}$ for $\mathrm{Zn}$ [6] decline for growing temperature in a similar way, see Fig. 2, and the temperature behavior of the

* e-mail: a.czachor@ncbj.gov.pl

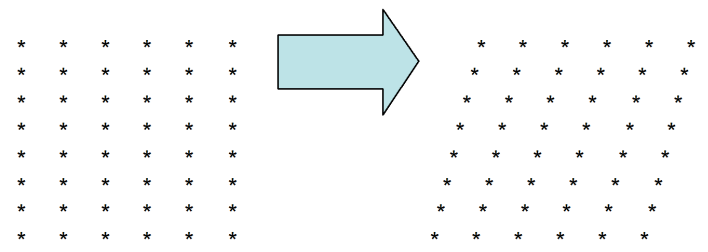

Fig. 1. Example of the macroscopic shear deformation (a part of the long-wave transverse acoustic normal mode vibration) of the $2 \mathrm{D}$ square crystal structure.

elastic constants related to shear deformations $-c_{44}$, $\left(c_{11}-c_{12}\right) / 2$ - is not much different from the behavior of the others. We show below, however, that due to the lowest value of the transverse sound velocity $\left(s_{i j} \approx\left(c_{i j}\right)^{1 / 2}\right)$ it is the transverse acoustic phonon mode that could be particularly effective in its structure disruptive power. This hypothesis is investigated within the quasi-harmonic theory of hot metal crystal, assuming two-body ion-ion interaction.

\section{Two-body potentials for metals}

Application of the concept of two-body potentials of the ion-ion interaction has, in the theory of condensed phase, a long tradition [7]. It has turned out to be useful for understanding and predicting many physical phenomena, but its physical justification is rather intuitive than firm. Nevertheless, in the $a b$ initio theory of metals one indeed arrives in the Hartree approximation at the phonon dynamical matrix $D_{\boldsymbol{q}}$ written in terms of the Fourier transforms of an ion-ion potential $V_{k} \equiv V(\boldsymbol{k})$, see e.g. [8]:

$$
\begin{aligned}
& D_{\boldsymbol{q}}^{\alpha \alpha^{\prime}}=\sum_{\kappa}\left[(\boldsymbol{q}+\boldsymbol{\kappa})_{\alpha}(\boldsymbol{q}+\boldsymbol{\kappa})_{\alpha^{\prime}} V(\boldsymbol{q}+\boldsymbol{\kappa})\right. \\
& \left.-\kappa_{\alpha} \kappa_{\alpha^{\prime}} V(\kappa)\right]
\end{aligned}
$$

where $\boldsymbol{q}$ is the phonon wave vector, the sum goes over 


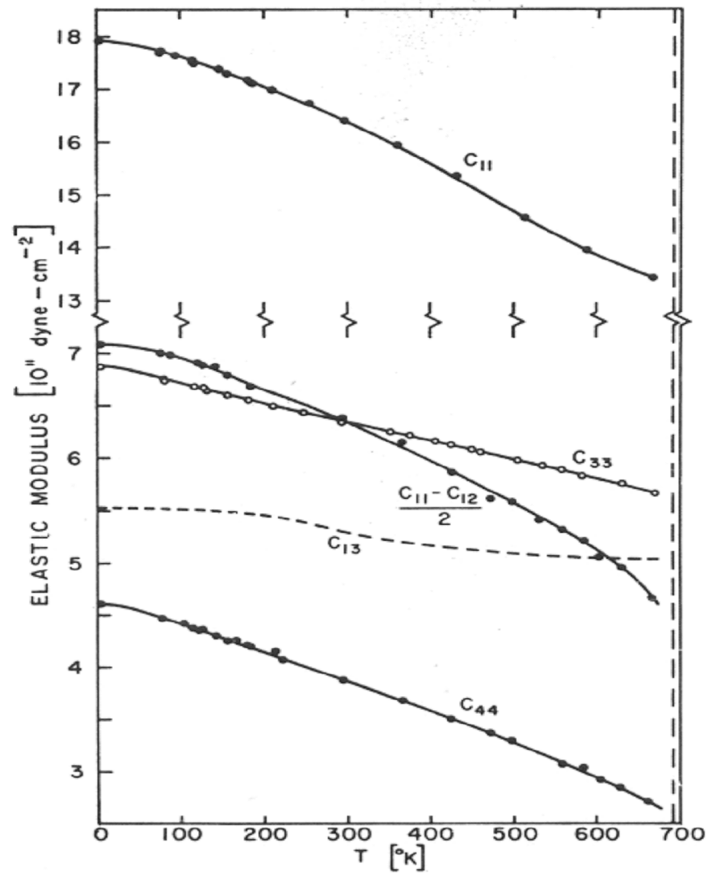

Fig. 2. Elastic constants of $\mathrm{Zn}$ as a function of temperature, according to Huntington [6]. $c_{44}$ and $\left(c_{11}-c_{12}\right) / 2$ are elastic constants for shear deformations.

the reciprocal lattice vectors $\kappa$, and

$$
\begin{aligned}
& V_{k}=\frac{(Z e)^{2}}{k^{2}}\left[1+\frac{k^{2} w^{2}(k)}{4 \pi Z^{2} e^{2}} \chi(k)\right], \\
& \chi(q)=\frac{\tilde{\chi}(q)}{1-\frac{4 \pi e^{2}}{q^{2}} \tilde{\chi}(q)}, \\
& \tilde{\gamma}(\boldsymbol{q})=\sum_{k} \frac{f\left(E_{k}-\mu\right)-f\left(E_{k+q}-\mu\right)}{E_{k}-E_{k+q}} .
\end{aligned}
$$

Here $w(k)$ is the ion-electron pseudopotential, $E_{k}$ is the electron energy band, $\mu$ is the Fermi energy and $Z$ is the electric charge of ion. The function $V_{k}$ can be treated as the Fourier transform of a certain two-body potential $V(r)$ :

$$
V_{k}=\frac{1}{V} \int V(\boldsymbol{r}) \exp (\mathrm{i} \boldsymbol{k} \cdot \boldsymbol{r}) \mathrm{d}^{3} r .
$$

We quote these $a b$ initio formulae only to set a firm ground for our relying on the ion-ion potential concept in description of metals near the melting temperature $T_{\mathrm{m}}$, but in the foregoing our argument will be phenomenological.

\section{Outline of the quasi-harmonic theory of lattice vibrations}

To represent the idea of the quasi-harmonic lattice dynamics theory let us first remind the standard harmonic Hamiltonian of a monoatomic crystal, valid for the small amplitudes $u_{l}$ of atomic vibrations [8]:

$$
H_{0}=\frac{1}{2 M} \sum_{l} p_{l}^{2}+\frac{1}{2} \sum_{l \neq l^{\prime}} \Phi_{l l^{\prime}}^{\alpha \alpha^{\prime}} u_{l}^{\alpha} u_{l^{\prime}}^{\alpha^{\prime}},
$$

where the force constants are given in terms of the ionion potential $V(r)$ :

$$
\Phi_{l l^{\prime}}^{\alpha \alpha^{\prime}}=-\left.\frac{\partial^{2} V(r)}{\partial r_{\alpha} \partial r_{\alpha^{\prime}}}\right|_{r=\left|\boldsymbol{l}-\boldsymbol{l}^{\prime}\right|} .
$$

Normal-mode vibration frequencies are the eigenvectors of this force constant matrix, while its eigenvalues are the (squared) normal mode frequencies $\omega_{j q}$, where $j$ is the so-called phonon branch label and $\boldsymbol{q}$ is the phonon wave vector. The condition $\omega_{j q}^{2}>0$ is the dynamical stability condition for the crystal.

To approach the melting phenomenon we have to allow for large atomic displacements, being at the root of the crystal structure destabilization. This can be systematically done using the Green function formalism of Zubarev [9]. Let us remind here that the Heisenberg operator $A(t)$ is defined as:

$$
A(t) \equiv \exp (\mathrm{i} H t) A \exp (-\mathrm{i} H t)
$$

and the equation of motion for such operator is

$$
\mathrm{i} \frac{\mathrm{d} A}{\mathrm{~d} t}=[A, H] \text {. }
$$

We derive now in the usual way the equation of motion for the (double-time retarded) displacementdisplacement Green function (GF) being a generalization of the correlation function of atomic displacements $\left\langle u_{l}^{\alpha}(t), u_{l^{\prime}}^{\alpha^{\prime}}\left(t^{\prime}\right)\right\rangle_{T}$ :

$$
\begin{aligned}
& G_{l l^{\prime}}^{\alpha \alpha^{\prime}}\left(t-t^{\prime}\right) \equiv\left\langle\left\langle u_{l}^{\alpha}(t), u_{l^{\prime}}^{\alpha^{\prime}}\left(t^{\prime}\right)\right\rangle\right\rangle \\
& \quad=-\mathrm{i} \theta\left(t-t^{\prime}\right)\left\langle\left[u_{l}^{\alpha}(t), u_{l^{\prime}}^{\alpha^{\prime}}\left(t^{\prime}\right)\right]\right\rangle_{T},
\end{aligned}
$$

where the thermal average of operator $X$ has the form

$$
\langle X\rangle_{T}=\frac{\operatorname{Tr}\left(\mathrm{e}^{-\beta H} X\right)}{\operatorname{Tr}\left(\mathrm{e}^{-\beta H}\right)},
$$

the $H$ being the Hamiltonian of the system.

Using commutation relations $\left[u_{l}^{\alpha}, p_{l^{\prime}}^{\alpha^{\prime}}\right]=\mathrm{i} \delta_{l l^{\prime}}^{\alpha \alpha^{\prime}}$ and the above harmonic Hamiltonian we obtain as the standard harmonic equation of motion in the GF formulation

$$
M\left(\frac{\mathrm{d}}{\mathrm{d} t}\right)^{2} G_{l l^{\prime}}^{\alpha \alpha^{\prime}}(t)=-\delta(t) \delta_{l l^{\prime}}^{\alpha \alpha^{\prime}}-\sum_{l^{\prime \prime} \alpha^{\prime \prime}} \Phi_{l l^{\prime \prime}}^{\alpha \alpha^{\prime \prime}} G_{l^{\prime \prime} l^{\prime}}^{\alpha^{\prime \prime} \alpha^{\prime}}(t) .
$$

It follows from the definition (7) of the force constants that to learn about the dynamics of crystal in case of small vibrations it is sufficient to know the curvatures of ion-ion potential $V(r)$ at the lattice vector positions $\boldsymbol{l}$.

At elevated temperatures the atomic displacements are large, so the atoms are experiencing the shape of the potential $V(r)$ at all values of inter-atomic distances. To allow such anharmonicity to show up in the harmonic theory scheme we assume the Hamiltonian in its full form

$$
H=\frac{1}{2} \sum_{l} p_{l}^{2}+\frac{1}{2} \sum_{l \neq l^{\prime}} V\left(\boldsymbol{l}-\boldsymbol{l}^{\prime}+\boldsymbol{u}_{l}-\boldsymbol{u}_{l^{\prime}}\right)
$$

with the ion-ion interaction potential $V(r)$ having a well determined Fourier transform $V_{k}$ such as in Eq. (5). Us- 
ing again the commutation relations one arrives in the above way at the more general form of the equation of motion for the displacement-displacement Green function $\boldsymbol{G}\left(t-t^{\prime}\right)$ :

$$
\begin{aligned}
& M\left(\mathrm{i} \frac{\mathrm{d}}{\mathrm{d} t}\right)^{2} G_{l l^{\prime}}^{\alpha \alpha^{\prime}}\left(t-t^{\prime \prime}\right) \\
& \quad=\delta\left(t-t^{\prime}\right) \delta_{l l^{\prime}}^{\alpha \alpha^{\prime}}+\sum_{k} \mathrm{i} k^{\alpha} V(k) \sum_{l^{\prime \prime}} \exp \left(\mathrm{i} \boldsymbol{k}\left(\boldsymbol{l}-\boldsymbol{l}^{\prime \prime}\right)\right) \\
& \quad \times\left\langle\left\langle\exp \left(\mathrm{i} \boldsymbol{k} \cdot\left(\boldsymbol{u}_{l}-\boldsymbol{u}_{l^{\prime \prime}}\right)\right), u_{l^{\prime}}^{\alpha^{\prime}}\left(t^{\prime}\right)\right\rangle\right\rangle .
\end{aligned}
$$

We can see that now on the right hand side (rhs) there is a new Green function, different from and more complex than the initial $\boldsymbol{G}\left(t-t^{\prime}\right)$. As compared to the equations of motion of harmonic theory (12) we can see that when one deals with full potential $V(r)$, the equations of motion for the GF become nonlinear. It means that the displacement-displacement correlations depend now in general on the higher-order correlation functions of more complex structure and physical content, than these contained in harmonic $\boldsymbol{G}\left(t-t^{\prime}\right)$. One can set equation of motion for this new GF, but the result will again contain even more sophisticated GFs. Besides, even if this process is finally terminated, the results are mathematically more complex than the results of the linear theory. This would need developing a new intuition and new calculation methods in the evaluation procedures for the physical quantities derivable from them.

There is in physics a practical exodus from such nonlinearity headache - a wise linearization of the equations of motion. In such case it is often possible to arrive at the approximate equations of the linear form, but with extra information somehow encoded in the parameters of these new linear equations.

In the present case, instead of sophisticated true solutions we shall purposefully return in the above way to the formulae of old harmonic theory, depending via new parameters on some extra features of the potential - on temperature and possibly on external forces. Of course, the new formulae should encompass the old ones as limiting case.

To do the linearization one postulates here an approximate "proportionality", being the essence of the quasi-harmonic approach to the theory of lattice vibrations $[10,11]$ :

$$
\begin{aligned}
& \left\langle\left\langle\exp \left(\mathrm{i} \boldsymbol{k} \cdot\left(\boldsymbol{u}_{l}-\boldsymbol{u}_{l^{\prime \prime}}\right)\right), u_{l^{\prime}}^{\alpha^{\prime}}\left(t^{\prime}\right)\right\rangle\right\rangle \\
& \approx \gamma\left\langle\left\langle\mathrm{i} \boldsymbol{k} \cdot\left(\boldsymbol{u}_{l}-\boldsymbol{u}_{l^{\prime \prime}}\right)(t), u_{l^{\prime}}^{\alpha^{\prime}}\left(t^{\prime}\right)\right\rangle\right\rangle \\
& \equiv \gamma \mathrm{i} k^{\alpha^{\prime \prime}}\left(\boldsymbol{G}_{l l^{\prime}}^{\alpha^{\prime \prime} \alpha^{\prime}}-\boldsymbol{G}_{l^{\prime \prime} l^{\prime}}^{\alpha^{\prime \prime} \alpha^{\prime}}\right)\left(t-t^{\prime}\right)
\end{aligned}
$$

with the proportionality factor $\gamma$ to be wisely determined now.

Let us remind the notion of "moments" of any GF, such as $\langle\langle A, B\rangle\rangle$. These are characteristic numbers for them: the first moment is $\langle[[A, H], B]\rangle_{T}$, the second is $\langle[[[A \cdot H], H,], B]\rangle_{T}$, etc. To impose physics on the above proportionality assumption we request that first non-vanishing moments of both sides must be equal. It works here with the "first moment" and in this way we have

$$
\begin{aligned}
\gamma & =\left\langle\exp \left(\mathrm{i} \boldsymbol{k} \cdot\left(\boldsymbol{u}_{l}-\boldsymbol{u}_{l^{\prime}}\right)\right)\right\rangle_{T} \\
& \approx \exp \left(-(1 / 2)\left\langle\left(\boldsymbol{k} \cdot\left(\boldsymbol{u}_{l}-\boldsymbol{u}_{l^{\prime}}\right)\right)^{2}\right\rangle_{T}\right)
\end{aligned}
$$

The second equality on the rhs is true in harmonic theory [8].

\section{Effective ion-ion quasi-potential}

In this way there appears in the quasi-harmonic-theory formula (14) a new form of the Fourier transform of ionion "potential" - the temperature-dependent effective quasi-potential

$$
\begin{aligned}
\tilde{V}_{k} & =V_{k} \exp \left(-(1 / 2)\left\langle\left(\boldsymbol{k} \cdot\left(\boldsymbol{u}_{l}-\boldsymbol{u}_{l^{\prime}}\right)\right)^{2}\right\rangle_{T}\right), \\
\boldsymbol{l} & \neq \boldsymbol{l}^{\prime} .
\end{aligned}
$$

The quotation mark reminds that the Fourier retransformed object $\tilde{V}(r)$, being dependent on the lattice vectors $\boldsymbol{l}, \boldsymbol{l}^{\prime}$, differs basically from the potential $V(r)$. In principle it is a different function for every pair of atoms $\left(\boldsymbol{l}, \boldsymbol{l}^{\prime}\right)$. This is the price we pay for enforcing the impact of anharmonicity upon the shoulders of the harmonic theory.

For this new correlation function in Eq. (17) we get in the harmonic theory for crystals of regular structure [8]:

$$
\begin{aligned}
& \left\langle\left(\mathrm{i} \boldsymbol{k} \cdot\left(\boldsymbol{u}_{l}-\boldsymbol{u}_{l^{\prime}}\right)\right)^{2}\right\rangle_{T} \\
& =-\frac{2 h k^{2}}{3 N M} \sum_{q j} \frac{\sin ^{2}\left(\boldsymbol{q} \cdot\left(\boldsymbol{l}-\boldsymbol{l}^{\prime}\right) / 2\right)}{\omega_{q j}} \operatorname{coth}\left(\frac{h \omega_{q j}}{2 k_{\mathrm{B}} T}\right) \\
& \underset{T \rightarrow \infty}{\longrightarrow}-d_{l-l^{\prime}} k^{2}
\end{aligned}
$$

where $d_{l-l^{\prime}}$ is a certain atom-atom correlation factor. It follows that it depends just on the relative position vector $\boldsymbol{l}-\boldsymbol{l}^{\prime}$ of the pair. For the present purpose it is sufficient to estimate the factor $d_{l}$ within the dispersion-less Debye model of dynamics: $\omega_{q \text { long }}=s_{l} q, \omega_{q \mathrm{tr}}=s_{\mathrm{tr}} q$. On the basis of experimental data one can roughly take for the sound velocities $s_{\text {long }}=2 s_{\mathrm{tr}}$. At high temperatures we can put $\operatorname{coth}(x) \longrightarrow_{x \rightarrow 0} 1 / x$. Having taken the angular average one obtains, with the notation $|\boldsymbol{l}|=l$ :

$$
d_{l} \approx \frac{2 k_{\mathrm{B}} T}{3 N M}\left(s_{l}^{-2}+s_{\mathrm{tr}}^{-2}\right) \sum_{q<k_{\mathrm{D}}} \frac{\sin ^{2}(\boldsymbol{q} \cdot \boldsymbol{l})}{q^{2}} \approx B_{l} \frac{T}{s_{\mathrm{tr}}^{2}} .
$$

At large distance $l$ we have the asymptotic value

$$
B_{l} \approx \frac{k_{\mathrm{B}}}{4 M n}\left(k_{\mathrm{D}}-\mathrm{O}\left(\frac{\pi}{4|\boldsymbol{l}|}\right)\right), \quad \boldsymbol{l} \neq 0,
$$

where $n=N / V=k_{\mathrm{D}}^{3} /\left(6 \pi^{2}\right)$.

The plot of the $d_{l}$ vs. $l$, Fig. 3 , shows that already at the nearest neighbor distance its value is close to the asymptotic value, so for $\boldsymbol{l} \neq 0$ we can approximately treat it as a constant number $d$. In this way we approximately return to the legitimate sense of the ion-ion potential. The Fourier transform of the effective ion-ion potential: it is the initial form multiplied with the Gaussian factor [8]: 


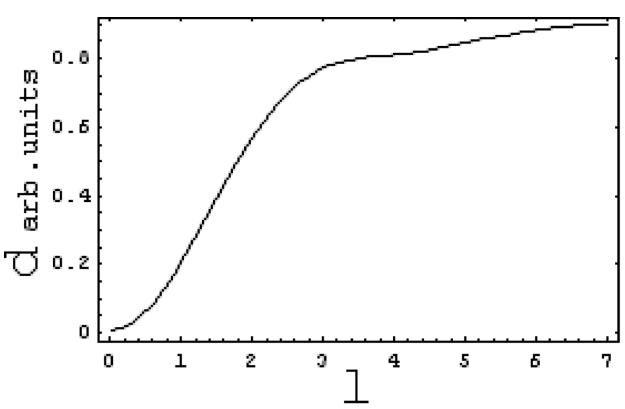

Fig. 3. Correlation factor $d_{l}$, Eq. (19), as a function of the lattice vector length under conditions given in text, evaluated numerically.

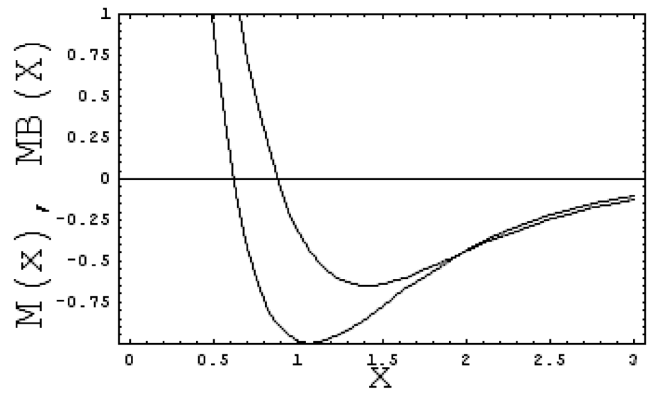

Fig. 4. Example of the temperature-induced modification of the effective ion-ion potential - the Morse potential $M(x)$ (lower curve) and its form $M B(x)$, "broadened" by multiplying the Fourier transform of $M(x)$ by a Gaussian factor, Eq. (21). Let us note the up and right shift of the minimum position and smaller curvature at minimum in the $M B(x)$, as compared to the $M(x)$.

$$
\tilde{V}_{k}=V_{k} \exp \left(-d k^{2}\right) \equiv V_{k} \Delta_{k} .
$$

Here function $\Delta_{k}$ describes the temperature induced "softening" (broadening) of the ion-ion interaction, which determines the lattice dynamics. We can see in Eq. (21) that the effective-potential Fourier transform at growing $T$ gets at large $k$ 's suppressed, thus the potential $V(r)$ becomes more smooth, "softer". This is illustrated in Fig. 4, using the Morse potential.

To pursue the impact of temperature on the transverse acoustic wave propagation we shall now use it in the phonon dynamical matrix.

\section{Transverse acoustic phonon softening as a precursor of melting}

Let us determine the impact of macroscopic shear deformation on the stability of crystals. As the case representative and simple let us take the propagation of long-wave $y$-polarized transverse oscillation along the direction $q_{x}$ parallel to [100] in the regular fcc metal structure. Following the phonon dynamical matrix, Eq. (1), phonon dispersion curve of the harmonic theory of vibrations can be written as follows [7]:

$$
M \omega_{T}^{2}\left(q_{x}\right)=D_{q_{x}}^{y y}=N \sum_{\kappa \neq 0} \kappa_{y}^{2}\left[V\left(\left|\boldsymbol{\kappa}+\boldsymbol{q}_{x}\right|\right)-V(\kappa)\right] .
$$

Let us note that due to the overall electric neutrality there is no $\kappa=0$ contribution in the sum (it appears in the dispersion relation for the longitudinal mode). On expanding Eq. (22), for small $q_{x}$ we obtain

$$
M \omega_{T}^{2}\left(q_{x}\right)=\frac{N}{2} q_{x}^{2} \sum_{\kappa \neq 0} \kappa_{x}^{2} \kappa_{y}^{2} / \kappa^{2} V^{\prime \prime}(\kappa) .
$$

Propagation of such long wave requires $\omega_{T}^{2}>0$, thus a domination of $V^{\prime \prime}(\kappa)>0$ terms in the sum. We want to include here the effect of growing temperature, i.e. of large atomic displacements. Having in mind the above developments, in Eq. (23) we shall use instead of $V_{k}$ its temperature modified form $\tilde{V}_{k}=V_{k} \Delta_{k}$, Eq. (21). Differentiation in $\tilde{V}_{k}^{\prime \prime}$ concerns both $V_{k}$ and $\Delta_{k}$ functions, but the Gaussian contribution represents the strongest variation and as a reasonable approximation we take as the dominant term: $\tilde{V}_{k}^{\prime \prime} \approx V_{k}^{\prime \prime} \Delta_{k}$. We shall concentrate on the transverse acoustic phonon curve $\omega_{\mathrm{tr}}\left(q_{x}\right)=s_{\mathrm{tr}} q_{x}$. On inserting it into the Eq. (23) we obtain the equation linking the transverse sound velocity $s_{\mathrm{tr}}$ to temperature $T$ and to some features of the Fourier transform of the ion-ion potential

$$
\begin{aligned}
& M s_{\mathrm{tr}}^{2}=\frac{1}{2} \exp \left(-B \frac{T}{s_{\mathrm{tr}}^{2}}\right) \sum_{\kappa \neq 0} \kappa_{x}^{2} \kappa_{y}^{2} / \kappa^{2} V^{\prime \prime}(\kappa) \\
& \equiv D \exp \left(-B \frac{T}{s_{\mathrm{tr}}^{2}}\right),
\end{aligned}
$$

where $D$ represents the "force" components essential for the transverse acoustic wave propagation. One can solve it with respect to $T$ (from now on symbol $s$ stands for $s_{\text {tr }}$ )

$$
T=s^{2}[\ln (D / M)-2 \ln (s)] / B .
$$

This is the main result of this paper - the equation showing the dependence of transverse sound velocity on temperature. One needs $D>0$ to have $s>0$ in a reasonable range of temperatures. At $T=0$ the velocity is the highest, $s_{\max }=(D / M)^{1 / 2}$. In the dimensionless units for the sound velocity $S=s / s_{\max }$ and for the temperature $\tau \equiv B T / s_{\mathrm{m}}^{2}$, the equation can be written as

$$
\tau=-2 S^{2} \log S, \quad 0<S<1 .
$$

Figure 5 and derived from it Fig. 6 show the corresponding plot. Slightly below $S=1$ the $\tau$ goes as $2(1-S)$. On looking for maximum of $\tau$ vs. $S$ we obtain $S_{\text {cr }}=\mathrm{e}^{-1 / 2}=0.606531$ and finally as the highest temperature at which the solution exist $\tau_{\text {cr }}=\mathrm{e}^{-1}$ or

$$
T_{\text {cr }}=0.367879 \frac{D}{M B} \text {. }
$$

At $T>T_{\text {cr }}$ Eq. (26) has not a real (or realistic) solution - the dynamic stability of the system disappears. The interpretation is that when one approaches this temperature from below, the medium reaches such high level of deformation and non-uniformity that it cannot support the propagation of the transverse sound wave. In fact 


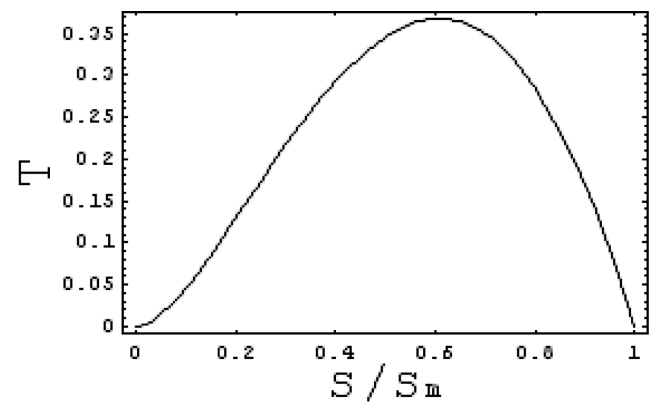

Fig. 5. Transverse acoustic sound velocity $S$, normalized to its maximum value at $T=0$, as a function of the crystal temperature $T$ - a direct plot of Eq. (26). The $S$-range from $S=0$ to the maximum of $T(S)$ has no physical meaning.

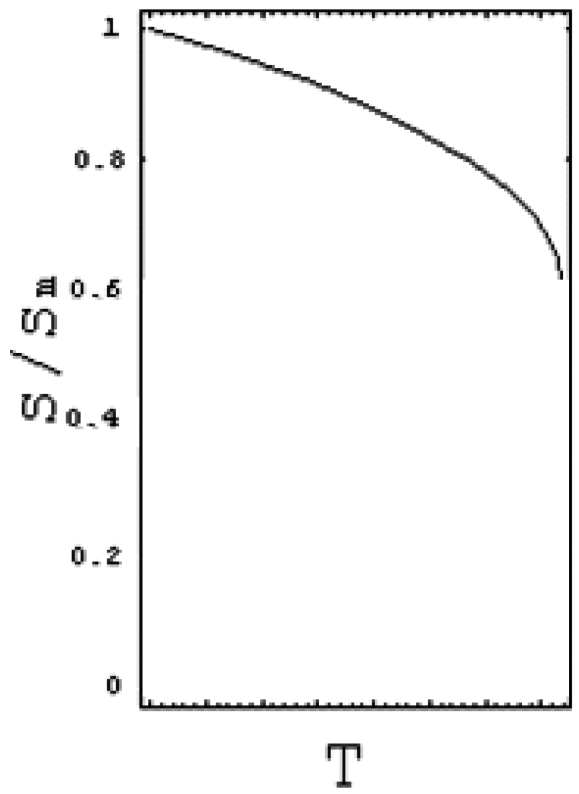

Fig. 6. Decline of the transverse sound velocity $S$ normalized to its maximum value $S_{\mathrm{m}}$ vs. temperature $T$, within the quasi-harmonic approximation, plotted according to Eq. (26). This picture appears after the axes inversion and the elimination of the unphysical part of the plot shown in Fig. 5.

this is a shear deformation, characteristic to the propagation of the transverse acoustic phonon oscillation. We emphasize that such special, perhaps pivotal, role of the acoustic transverse oscillation is a consequence of the low velocity of the transverse sound wave, resulting at a given temperature of the crystal in a large amplitude of crystal oscillation related to it.

For its verification the model shown here needs experimental data on transverse sound velocity, crystal structure and linear expansion close to the melting temperature for several metal systems.

\section{Summary}

Within the quasi-harmonic approximation of lattice dynamics of metals we have shown that the temperature decline of the transverse sound velocity can manifest itself in such a large deformation of crystal, that it ceases to support the related shear oscillation, thus leading to lattice disordering and melting of the structure. This phenomenon is a consequence of the ion-ion effective potential broadening due to temperature-enhanced vibrations, and can be described by a simple equation involving temperature and transverse sound velocity.

\section{References}

[1] A.R. Ubbelohde, Melting and Crystal Structure, Clarendon Press, Oxford 1965.

[2] S.M. Stishov, Sov. Phys. Usp. 11, 816 (1969).

[3] D. Stroud, N.W. Ashcroft, Phys. Rev. B 5, 371 (1972).

[4] F.A. Lindemann, Z. Phys. 11, 609 (1910).

[5] M. Ross, Phys. Rev. 184, 233 (1969).

[6] G.A. Alers, J.R. Neighbours, in: The Elastic Constants of Crystals, Ed. B.H. Huntington, Academic Press, New York and London 1958, Vol. 7, p. 109.

[7] H.R. Schober, in: New Concepts in the Physics of Phonons, Ed. A. Czachor, Report INR 1743/II/PS/A, Warszawa 1978, p. 26.

[8] A. Czachor, Atomic Vibrations in Solids, PWN, Warszawa 1982 (in Polish)

[9] D.N. Zubarev, Sov. Phys. Usp. 3, 320 (1960).

[10] H.M. Plakida, T. Siklos, Acta Phys. Hung. 26, 387 (1969).

[11] K. Parliński, Physica 51, 299 (1971). 